OPEN ACCESS

Edited by:

Zijun Wang,

The Rockefeller University,

United States

Reviewed by:

Kurt B. Pruner

University of Washington,

United States

Javier Ibarrondo,

UCLA Health System, United States

*Correspondence:

Alexander V. Filatov avfilat@yandex.ru

Specialty section:

This article was submitted to Immunological Memory,

a section of the journal

Frontiers in Immunology

Received: 21 December 2021 Accepted: 07 February 2022

Published: 24 February 2022

Citation:

Byazrova MG, Kulemzin SV, Astakhova EA, Belovezhets TN,

Efimov GA, Chikaev AN.

Kolotygin 1O, Gorchakov AA,

Taranin AV and Filatov AV (2022)

Memory B Cells Induced by

Sputnik V Vaccination Produce

SARS-CoV-2 Neutralizing Antibodies

Upon Ex Vivo Restimulation.

Front. Immunol. 13:840707.

doi: 10.3389/fimmu.2022.840707

\section{Memory B Cells Induced by Sputnik V Vaccination Produce SARS-CoV-2 Neutralizing Antibodies Upon Ex Vivo Restimulation}

Maria G. Byazrova ${ }^{1,2}$, Sergey V. Kulemzin ${ }^{3}$, Ekaterina A. Astakhova ${ }^{1,2}$,

Tatyana N. Belovezhets ${ }^{3}$, Grigory A. Efimov ${ }^{4}$, Anton N. Chikaev ${ }^{3}$, llya O. Kolotygin ${ }^{3}$, Andrey A. Gorchakov ${ }^{3}$, Alexander V. Taranin ${ }^{3}$ and Alexander V. Filatov ${ }^{1,2 *}$

\footnotetext{
1 Laboratory of Immunochemistry, National Research Center Institute of Immunology, Federal Medical Biological Agency of Russia, Moscow, Russia, 2 Department of Immunology, Faculty of Biology, Lomonosov Moscow State University, Moscow, Russia, ${ }^{3}$ Laboratory of Immunogenetics, Institute of Molecular and Cellular Biology, Siberian Branch of the Russian Academy of Sciences, Novosibirsk, Russia, ${ }^{4}$ Laboratory of Transplantation Immunology, National Research Center for Hematology, Moscow, Russia
}

The development of effective vaccines against SARS-CoV-2 remains a global health priority. Despite extensive use, the effects of Sputnik $V$ on B cell immunity need to be explored in detail. We performed comprehensive profiling of humoral and B cell responses in a cohort of vaccinated subjects $(n=22)$, and demonstrate that Sputnik vaccination results in robust $B$ cell immunity.

We show that B memory cell (MBC) and antibody responses to Sputnik $\vee$ were heavily dependent on whether the vaccinee had a history of SARS-CoV-2 infection or not. 85 days after the first dose of the vaccine, ex vivo stimulated MBCs from the vast majority of Sputnik $V$ vaccinees produced antibodies that robustly neutralized the Wuhan Spikepseudotyped lentivirus. MBC-derived antibodies from all previously infected and some of the naïve vaccine recipients could also cross-neutralize Beta (B.1.351) variant of SARS-CoV-2.

Virus-neutralizing activity of MBC-derived antibodies correlated well with that of the serum antibodies, suggesting the interplay between the MBC and long-lived plasma cell responses. Thus, our in-depth analysis of MBC responses in Sputnik $V$ vaccinees complements traditional serological approaches and may provide important outlook into future $B$ cell responses upon re-encounter with the emerging variants of SARS-CoV-2.

Keywords: memory B cells, Sputnik V vaccine, COVID-19, SARS-CoV-2, vaccination 


\section{INTRODUCTION}

Presently, the therapeutic options for COVID-19 patients remain limited, emphasizing the necessity of concerted mass vaccination campaigns to counteract the pandemic. An ideal vaccine must induce long-lasting protective cellular and humoral immunity, which should translate into reduced rates of infection and mortality. Importantly, an ideal vaccine should, in addition, retain activity against emerging viral lineages. Three anti-SARSCoV-2 vaccines, Moderna mRNA-1273, BioNTech BNT162b2, and Janssen Ad26.COV2.S, are now being extensively used around the world and have received the most public attention and validation $(1,2)$, while less is known about immunity after Sputnik V vaccination (3). Although humoral responses to Sputnik V have recently been reported for a limited number of study participants $(4,5)$, data regarding the B cell response in Sputnik V-vaccinated subjects are presently lacking. Clearly, these data are central to the comprehensive assessment of current vaccines (1), provide important clues to the development of new vaccines, and impact the epidemiological models of immunity.

In late 2020, multiple SARS-CoV-2 lineages were reported across the globe, of which Alpha (B.1.1.7), Beta (B.1.351), and Delta (B.1.617) are now referred to as variants of concern (VOCs) $(6,7)$. Beta and Delta display profound resistance to most of the approved highly potent neutralizing monoclonal antibodies, as well as to the polyclonal antisera induced by infection with the ancestral SARS-CoV-2 and by all the vaccines developed to date (8-11). It is generally believed that it was the emergence and rapid spread of VOCs that are largely responsible for the documented cases of SARS-CoV-2 reinfection $(12,13)$. Specifically, post-vaccination antisera from Moderna and BioNTech vaccinees were 6.5-40-fold less potent against the Beta VOC, but most typically neutralization was reduced 3-8-fold compared to that of the ancestral Wuhan-1 SARS-CoV-2 strain (14). So far, qualitative and quantitative data on the VOC neutralization by Sputnik V-induced antisera have been very limited $(4,15)$.

Notably, in contrast to Moderna mRNA-1273, BioNTech BNT162b2, and Janssen Ad26.COV2.S vaccines that were designed to present the SARS-CoV-2 Spike protein in its prefusion conformation, Sputnik V is based on a native Spike protein lacking such modifications. This, in turn, may underlie distinct immune responses, upon cross-platform comparisons, and warrants in-depth analysis (16). The level of virus binding and virus neutralizing serum antibodies is the most studied parameter of the B cell response to SARS-CoV-2 in both convalescents and vaccinated subjects. Much less is known about the antibodies that MBCs will secrete during the secondary immune responses. To address this question, quantification of the levels of RBD-specific and virus neutralizing antibodies in cultures of polyclonally ex vivo stimulated B cells is needed, as it may provide a measure of B memory cell immunity and predict the outcome of infection.

In this study, we aimed to determine i) whether Sputnik V vaccination is efficient in inducing durable memory B cell (MBC) immunity, ii) whether stimulated MBCs are capable of secreting virus-neutralizing antibodies, and if so, iii) whether Sputnikinduced serum and $\mathrm{MBC}$-derived antibodies are protective against one of the most neutralization-resistant SARS-CoV-2 viral variants Beta B.1.351 or not. To date, these issues have not been explored in detail and addressing these research gaps should be instrumental for the development of next-generation SARS-CoV-2 vaccines.

\section{RESULTS}

Twenty-two healthy subjects were recruited during the winter/ spring of 2021 to receive two doses of the Gam-COVID-Vac (Sputnik V) vaccine (Figures 1A, S1). The demographic characteristics of this cohort are provided in Table S1. The age of the volunteers ranged from 25 to 70 years (median age, 60.0 years; an interquartile range (IQR), 49.8-63.0 years; 64\% female). Five individuals recruited in October-November 2020 had experienced mild COVID-19 symptoms prior to vaccination (53-120 days). Although no virus-containing samples were available for these patients, it must be noted that the SARSCoV-2 viral lineage Alfa (B.1.1.7) was predominant in Moscow at that time and it was only in March 2021 that the first Betaassociated infections were reported in Moscow (15). Before vaccination, no nucleocapsid $(\mathrm{N})$ - or receptor-binding domain (RBD)-specific IgGs were detected in the sera of naïve individuals without prior COVID-19 symptoms. In contrast, all recovered recipients with self-reported COVID-19 symptoms had both $\mathrm{N}$ - and RBD-specific IgG prior to vaccination (Figure S2). In order to investigate the plasmablast and $\mathrm{MBC}$ responses, the following study design was used: blood samples were collected before vaccination (T0), one week after the first and second doses (T1 and T2, correspondingly), and on day 85 after the first dose (Figure 1A, Figure S1).

\section{Serum Antibody Responses to Sputnik V Vaccination}

Plasma samples from the vaccinated individuals were tested for IgG, IgA and IgM RBD-specific antibodies using ELISA. Longitudinal analysis of circulating serum antibodies showed that the levels of anti-RBD IgG and IgA increased markedly after vaccination (Figure 1B). In naive vaccine recipients, the RBD-specific IgG levels mainly increased after the second vaccine dose ( $\mathrm{T} 1$ vs. $\mathrm{T} 2, \mathrm{P}=0.0156)$. In recovered vaccine recipients, the levels of RBD-specific IgG were higher at baseline and displayed a more pronounced increase after the first vaccine dose (T0 vs. T1, $\mathrm{P}=0.0374$ ). Both in recovered and naïve vaccine recipients, the serum RBD-specific IgG levels achieved after the second dose remained stable until day 85. A similar trend was observed for RBD-specific IgA antibodies; however, the overall increase was not as strong. The anti-RBD IgM response in vaccinated individuals was low. The increase in RBD-specific IgM levels was most pronounced in naïve individuals at T2 (T0 vs. T2, P < 0.0001), consistent with the primary nature of their immune response. Taken together, these results are consistent with those of previous reports exploring antibody responses to mRNA vaccines $(1,17)$.

Next, we investigated if plasma from Sputnik V-vaccinated subjects, with or without prior COVID-19 history, was active in 


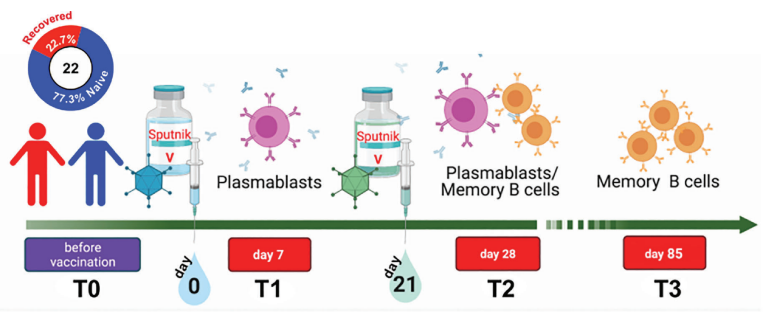

C

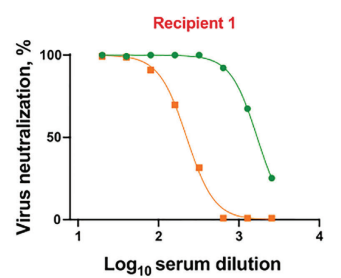

D

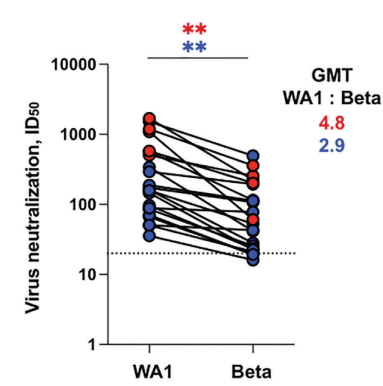

E

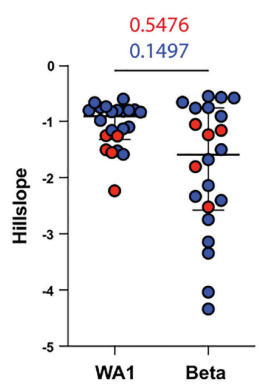

B
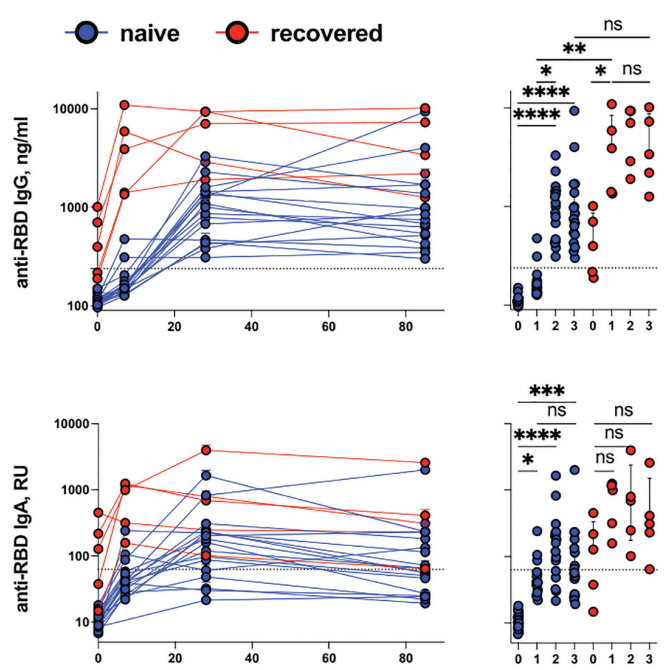

\begin{tabular}{llllllllll}
\hline & 1 & 1 & 1 & 1 & 3 & 0 & 1 & 2 & 3
\end{tabular}
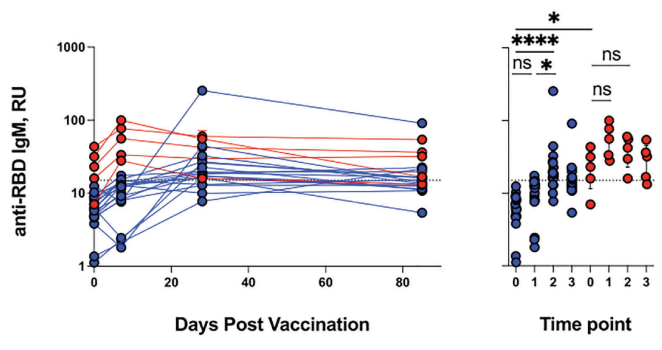

F
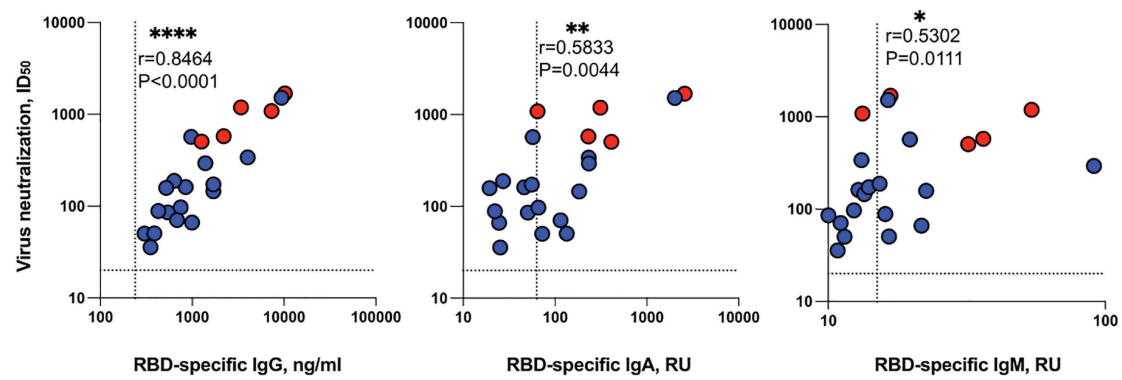

FIGURE 1 | Virus-binding and virus-neutralizing activity of sera from Sputnik V-vaccinated individuals. (A) Study design. (B) Serum anti-RBD IgG (top row), IgA (middle row) or IgM (bottom row) levels for all Sputnik V-vaccinated individuals, measured by ELISA. IgA and IgM levels are shown as a relative units (RU) against a standard convalescent serum. (C) Representative neutralization curves of vaccine-induced sera from one recovered and one naive individual at T3 time point.

(D) Paired analysis of neutralization titers $\left(\mathrm{ID}_{50}\right)$ against WA1 strain and Beta variant at $\mathrm{T} 3$ time point. (E) Analysis of hillslopes of virus neutralization curves for sera from vaccinated individuals. (F) Spearman's correlation between serum virus neutralization half-maximal inhibitory serum dilution ( $\left(\mathrm{ID}_{50}\right)$ values and the serum levels of anti-RBD IgG (left panel), IgA (middle panel) and IgM (right panel). Blue and red symbols indicate naïve $(n=17)$ and recovered $(n=5)$ participants. Symbols connected by solid lines represent time points considered for each individual. Data are presented as median \pm IQR. The dotted lines indicate the threshold for positivity. Statistics were calculated using Mann-Whitney test (comparisons between WA1 strain and Beta variant) or the Kruskal-Wallis test (comparisons between time points and naïve and recovered, ${ }^{*} \mathrm{P}<0.05,{ }^{* \star} \mathrm{P}<0.01,{ }^{* \star *} \mathrm{P}<0.001,{ }^{* \star * *} \mathrm{P}<0.0001$, ns, not significant. GMT, geometric mean titer; ID ${ }_{50}$, half-maximal inhibitory dilution; IQR, interquartile range; RBD, receptor-binding domain; $\mathrm{RU}$, relative units.

terms of virus neutralization against the wild-type SARS-CoV-2 strain WA1 and Beta VOC. To address this question, a SARSCoV-2 Spike-pseudotyped virus-neutralization test (pVNT) was used to analyze the sera collected at T3 (Figure 1C). Beta VOC is known to be one of the most neutralization-resistant viral variants (18) and encompasses multiple Spike substitutions (of which three, K417N, E484K, and N501Y, are in the RBD). All the 22 serum samples were able to neutralize WA1, although neutralization potency varied broadly with $\mathrm{ID}_{50}$ ranging from 36-1690 [geometric mean titer (GMT) 215, Figures 1C, D). 
Compared to the sera from the naïve group of vaccinees, samples obtained from the recovered group demonstrated significantly higher neutralization activity against both WA1 $\left(\mathrm{ID}_{50}\right.$ GMT 913 vs. $\left.149, \mathrm{P}=0.0019\right)$, and Beta $\left(\mathrm{ID}_{50} \mathrm{GMT}\right.$ 190.7 vs. $49.8, \mathrm{P}=0.0111$ ). Importantly, the GMT values for Beta were 2.9-fold lower than those for WA1 in naive recipients $(\mathrm{P}=$ 0.0038) (Figure 1D). All T3 sera from the five recovered subjects displayed 90\% neutralization of Beta at a 1:20 dilution (Figure S3). In contrast, in the naïve group, only one out of the total 17 samples (subject 19) displayed similar neutralization potency. Nonetheless, the undiluted sera could achieve $90 \%$ neutralization in all but one of the naïve samples. The neutralization activity in that exceptional sample (subject 22) could not be reliably measured. However, the neutralization titers we measured in the cohort of naïve Sputnik V vaccinees were significantly higher than those reported by Ikegame et al. (4). Specifically, they were three-fold higher for the WA1 Spike (ID ${ }_{50}$ GMT 149; 95\% confidence interval (CI) - 85.7-229.8) and seven-fold higher for Beta ( $\mathrm{ID}_{50}$ GMT 49.8; 95\% CI - 28.67-83.49). Overall, neutralizing activities of the sera against WA1 and Beta displayed pronounced positive correlation (Spearman's $r=$ 0.8647, P < 0.0001; Figure S4), a neutralization curve shape comparison did not reveal significant differences between the Hill slopes for WA1 and Beta $(\mathrm{P}=0.1497$ and $\mathrm{P}=0.5476$ for naïve and recovered individuals, respectively) (Figure 1E), indicating that the neutralization abilities of sera towards WA1 and Beta vary more in quantitative rather than in qualitative terms.

Finally, the levels of WA1 virus neutralization were strongly correlated with the level of RBD-specific IgG and modestly correlated with that of IgA and IgM (Spearman's $r=0.8464$, $\mathrm{P}<0.0001 ; \mathrm{r}=0.5833, \mathrm{P}=0.0044 ; \mathrm{r}=0.5302, \mathrm{P}=0.0111$, for IgG, $\operatorname{IgA}$, and $\operatorname{IgM}$, respectively) (Figure $\mathbf{1 F}$ ), suggesting that $\mathrm{IgG}$ antibodies are the most potent neutralizing component of the sera.

\section{Total and RBD-Specific Plasmablast Response}

One of the earliest manifestations of the $\mathrm{B}$ cell response is the emergence of circulating total and antigen-specific plasmablasts, which peak around the seventh day post-immunization (19). Plasmablasts were defined here as $\mathrm{CD}^{-} \mathrm{CD} 16^{-} \mathrm{CD} 19^{+} \mathrm{IgD}^{-}$ $\mathrm{CD} 27^{\text {hi }} \mathrm{CD} 38^{\text {hi }}$ cells (19) (Figure 2A, left panel). Before vaccination, the plasmablast frequencies were the same as those in normal donors but increased markedly after the primary immunization (T0 vs. $\mathrm{T} 1, \mathrm{P}=0.0076$ and $\mathrm{P}=0.0289$ for naïve and recovered individuals, respectively) (Figure 2B). Booster immunization resulted in an insignificant increase in the percentage of total plasmablasts compared to the baseline level. Positive plasmablast response was detected in $81 \%(17 / 21)$ of cases at T1 and only in $40 \%(8 / 20)$ of cases at T2. Subsequently, as can be seen from the measurements at T3, plasmablasts completely disappeared from the circulation.

The dynamics of SARS-CoV-2-specific plasmablasts during vaccination were of special interest to us. Since virus-neutralizing antibodies are known to predominantly target the RBD (8), when detecting antigen-specific $B$ cells, we focused on detecting RBDbinding $\left(\mathrm{RBD}^{+}\right)$cells. Antigen-specific plasmablasts were detected by double staining using RBD-PE and RBD-APC (Figure 2A, middle panel). As a negative control, we used the samples stained with an irrelevant PE-labeled protein Bet $\mathrm{v} 1$, which is the major birch allergen (Figure 2A, right panel) (none of the study participants had birch pollen allergy). Based on this negative control, the cut-off value for RBD-binding plasmablasts was set at $0.01 \%$. In contrast to COVID-19 patients sampled in the acute phase of the disease (20), the frequencies of $\mathrm{RBD}^{+}$ plasmablasts in vaccinated individuals were low (Figure 2C). The $\mathrm{RBD}^{+}$plasmablast response was detectable in 18 subjects (86\%) after the first dose of Sputnik V and only in eight participants $(40 \%)$ after the booster immunization. In only one subject from the recovered subgroup, it exceeded $1 \%$, which is still significantly lower than what was observed in patients with moderate COVID-19 (20, 21).

Since plasmablasts are antibody-secreting cells (ASCs), it is logical to detect and enumerate them using an enzyme-linked immunosorbent spot (ELISpot) assay. Representative ELISpot images of circulating Spike- and RBD-specific IgG ASCs are presented in Figure 2D. Historic control wells showed only rare spontaneous total ASCs and no SARS-CoV-2-specific ASCs. The magnitude of the IgG ASC response was the largest in the recovered group after the first vaccine dose, when RBD- and Sspecific IgG ASCs were detected in all vaccine recipients (median 69, IQR 27.33-772.8 and median 459, IQR 318-1439 for RBDand S-specific ASCs, respectively) (Figure 2E). In contrast, no RBD- or S-specific IgG ASCs were found in all but one naïve subject (recipient 8) after the first dose. After the second vaccination, 7 and 11 naive participants $(n=15)$ had RBDand S-specific IgG ASCs above the baseline, respectively. The fact that after the first vaccine dose, the frequencies of anti-RBD IgG ASCs were higher in SARS-CoV-2-recovered individuals than in individuals in the naïve group is consistent with the idea that, in the former cohort, this increase is due to the re-activation of MBCs. As for the IgA ASCs, the most notable difference was that both RBD- and S-specific ASCs were significantly overrepresented in the SARS-CoV-2-recovered (median 76, IQR 8.375-186.9 and median 62.22, IQR 19.75-394.2 for RBDand S-specific ASCs, respectively) vs naïve (median 1, IQR 1-4.5 and median 2.2, IQR 1-4 for RBD- and S-specific ASCs, respectively) individuals. The SARS-CoV-2-specific IgM responses mediated by the circulating ASCs were generally lower in their magnitude than the IgG responses and we did not observe significant differences between the naive and recovered samples, nor between the $\mathrm{T} 1$ and $\mathrm{T} 2$ time points.

Plasmablasts are a heterogeneous population of cells that are usually subdivided into early and later plasmablasts based on their ability to express the $\mathrm{B}$ cell receptor (BCR) on their surface and secrete antibodies (22). For the most part, plasmablasts express membrane bound BCR and simultaneously secrete antibodies. However, surface BCR expression is more a characteristic of early plasmablasts, while antibody-secreting capacity is more associated with later plasmablasts. Accordingly, we found a modest correlation between the 
A

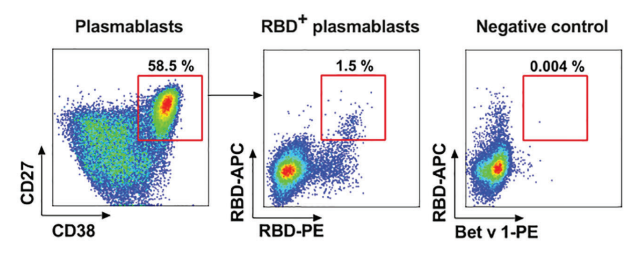

C
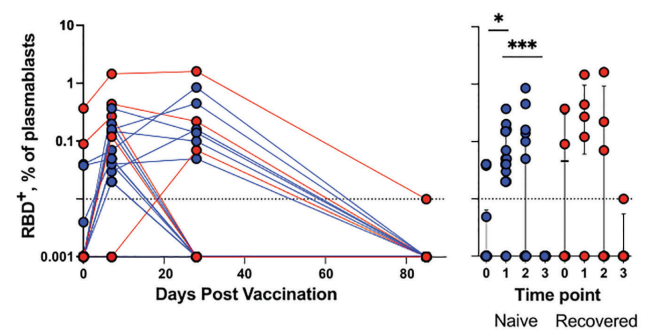

D

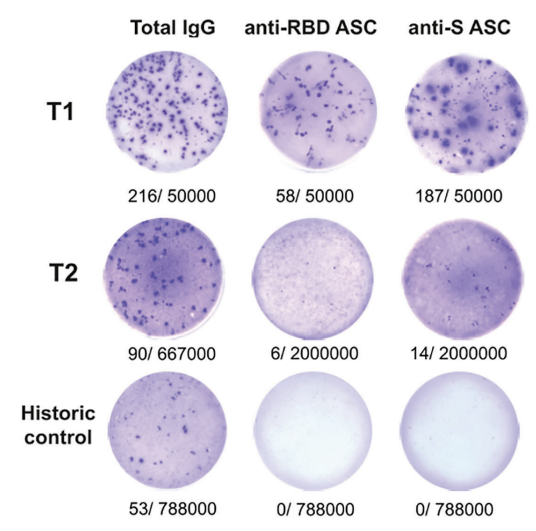

$\mathbf{F}$

T1

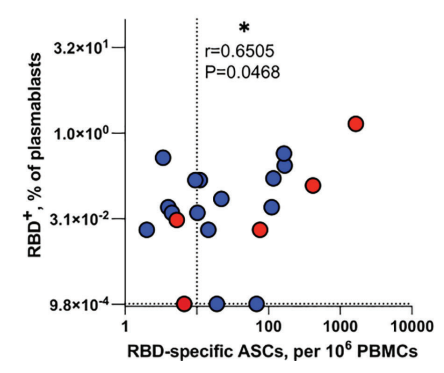

B
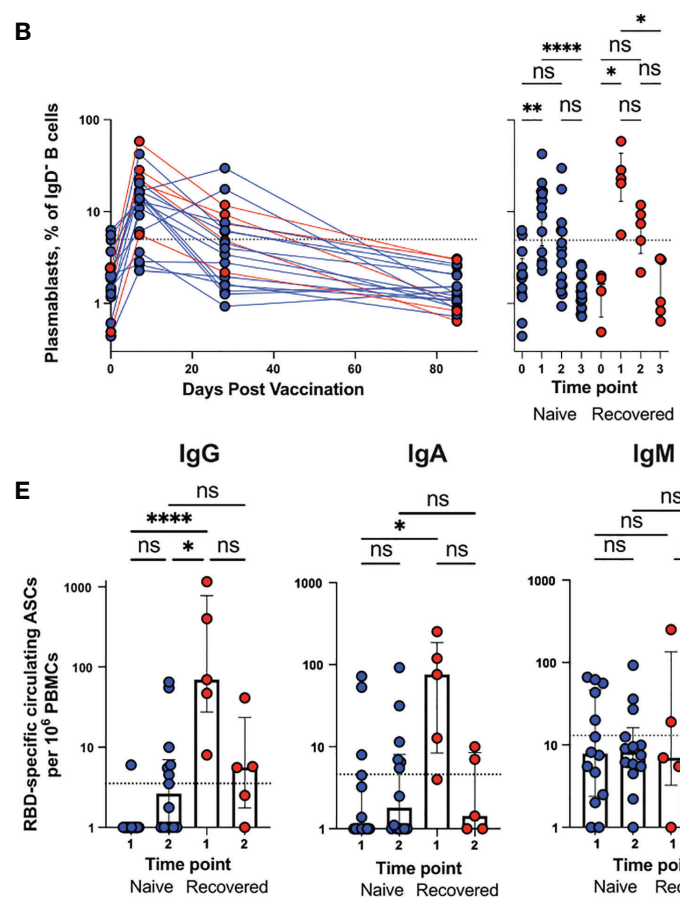

Naive Recovered
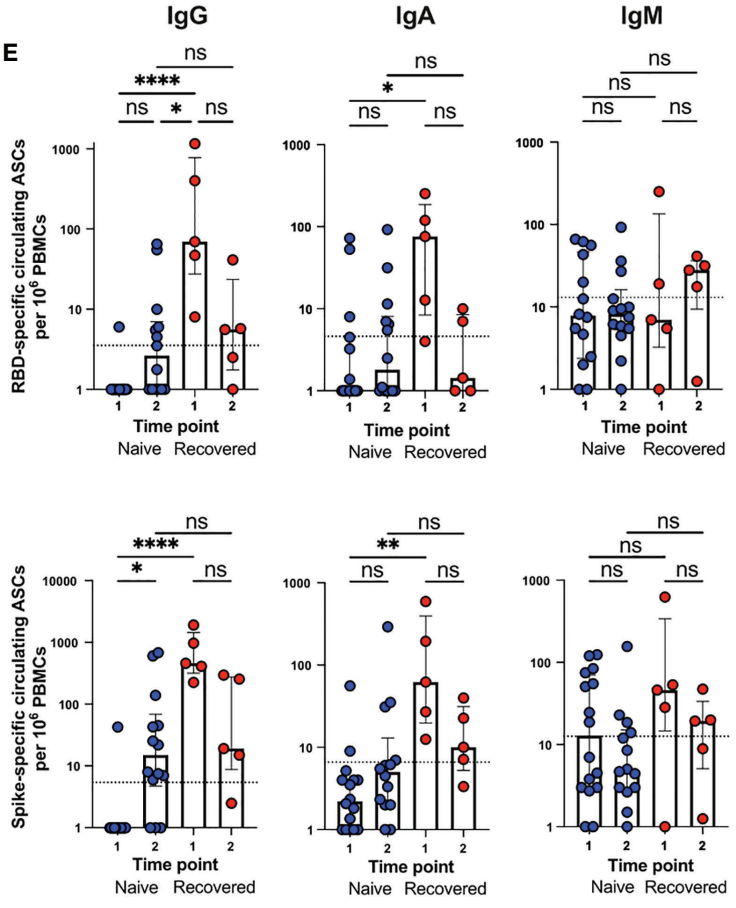

T2

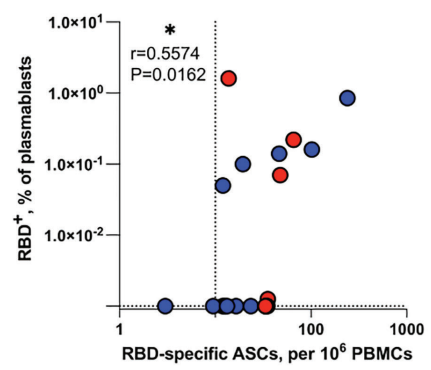

FIGURE 2 | Quantification of total and RBD-specific plasmablasts in blood samples from Sputnik V-vaccinated individuals. (A) Representative flow cytometry dot plots showing the gating strategy for measuring the percentage of total (left panel) and RBD ${ }^{+}$(middle panel) plasmablasts. As a negative control, sample stained with an irrelevant protein Bet $\mathrm{v} 1$ is shown (right panel). Numbers inside the plots indicate the percentage of events specific to respective gates. (B, C) Dynamic changes in total (B) and $\mathrm{RBD}^{+}$(C) plasmablast frequencies in samples collected at different time points. (D) Representative ELISpot images for measuring the frequencies of circulating total (left), anti-RBD (middle) or anti-S (right) IgG ASCs. Numbers below the wells represent the frequencies of ASCs relative to the total number of cells in the well. (E) Frequencies of circulating IgG (left column), IgA (middle column) or IgM (right column) ASCs specific for RBD (upper row) or S (bottom row) antigens per $10^{6}$ PBMCs collected from vaccinated individuals at different time points. The dotted lines indicate the threshold for positive antigen-specific ASC responses. (F) Spearman's correlation between RBD-specific (IgG + IgA + IgM) ASCs and the levels of RBD+ plasmablasts at T1 (left) and T2 (right) time points. Results are shown for individual samples (symbols) from naïve $(n=17)$ and recovered $(n=5)$ recipients. Data are presented as median \pm IQR. Asterisks indicate significant difference between groups determined using the Kruskal-Wallis test, ${ }^{\star} P<0.05,{ }^{\star \star} P<0.01,{ }^{* \star *} \mathrm{P}<0.001,{ }^{\star \star \star \star *} \mathrm{P}<0.0001$, ns, not significant. ACS, antibody-secreting cell; IQR, interquartile range; RBD, receptor-binding domain. 
frequencies of $\mathrm{RBD}^{+}$plasmablasts and RBD-specific Ig (IgG + $\operatorname{IgA}+\operatorname{IgM}$ ) circulating ASCs (Spearman's $\mathrm{r}=0.6505, \mathrm{P}=0.0468$ at $\mathrm{T} 1 ; \mathrm{r}=0.5574, \mathrm{P}=0.0162$ at T2) (Figure 2F).

\section{SARS-CoV-2-Specific Memory B Cell Response After Sputnik V Vaccination}

To investigate whether Sputnik V-vaccinated individuals developed antigen-specific MBCs, we used two complimentary approaches, namely, flow cytometry of RBD-binding circulating cells $(1,23,24)$ and quantification of SARS-CoV-2-specific ASCs induced by in vitro interleukin 21 (IL-21)/CD40L stimulation (20). The $\mathrm{RBD}^{+} \mathrm{MBC}$ were defined as $\mathrm{CD} 19^{+} \mathrm{CD} 27^{+} \mathrm{CD} 38^{-}$and double-positive for the fluorescently labeled RBD-PE and RBDAPC following exclusion of $\operatorname{IgD}^{+} \mathrm{B}$ cells (Figure 3A). The frequencies of $\mathrm{RBD}^{+} \mathrm{MBCs}$ measured at different time points are shown in Figure 3B. Even before vaccination, the recovered individuals had a noticeable number of $\mathrm{RBD}^{+} \mathrm{MBCs}$, which was higher than that of naive subjects $(\mathrm{P}=0.0362)$, and above the level of the negative control defined by staining with an irrelevant Bet $\mathrm{v} 1$ protein $(0.01 \%)$. Until day 85 , the level of $\mathrm{RBD}^{+} \mathrm{MBCs}$ in recovered individuals remained on average stable. In naïve individuals, baseline frequencies of $\mathrm{RBD}+\mathrm{MBCs}$ were observed at $\mathrm{T} 0$ and $\mathrm{T} 1$. At $\mathrm{T} 2, \mathrm{RBD}^{+} \mathrm{MBCs}$ were detected above the threshold in $37.5 \%$ of naïve individuals, displayed a further increase at $\mathrm{T} 3$ ( $\mathrm{T} 1$ vs. $\mathrm{T} 3, \mathrm{P}=0.0023$ ), and approached the level observed in recovered individuals.

Measurements of the SARS-CoV-2-specific circulating MBC numbers were supplemented with a more functional ELISpot assay. In contrast to plasmablasts, MBCs are resting cells and do not secrete antibodies without stimulation. For MBC activation and induction of antibody secretion, immunomagnetically purified B cells were stimulated in vitro with IL-21/CD40L. After stimulation for 7 days, the frequencies of S- and RBDspecific ASCs were evaluated using an ELISpot assay. As shown in the representative ELISpot images (Figure 3C), our protocol for polyclonal B cell activation was highly efficient and resulted in the secretion of both total and SARS-CoV-2-specific antibodies. In pre-pandemic control samples, the frequencies of SARS-CoV2-specific IgG ASCs were below 200 and 215 spots per million B cells were seeded in Spike- and RBD-coated wells. These values served as a cut-off for positivity. The MBC-derived ASC numbers were measured at two time points, 28 (T2) and 85 (T3) days after the first dose of the vaccine, when MBCs become detectable (T2) and undergo maturation (T3).

MBC-derived IgG ASCs displayed the strongest response. At $\mathrm{T} 2$, in all recovered (5/5) and in some naive subjects (7/17) the SARS-CoV-2-specific MBC-derived ASC numbers were above the baseline; however, recovered subjects had a higher level of ASCs than naïve subjects $(\mathrm{P}=0.034$ and $\mathrm{P}=0.0373$ for $\mathrm{RBD}$ - and $\mathrm{S}$-specific ASCs, respectively) (Figure 3D). At T3, the numbers of ASCs in recovered vaccinees remained stable, while in naïve subjects they increased 14-fold and approached the recovered group level (in the naïve group T2 vs. T3, $\mathrm{P}<0.0001$ and $\mathrm{P}=$ 0.0002 for RBD- and S-specific ASCs, respectively). On average, at the peak of the response, approximately 3,000 RBD-specific IgG ASCs per million B cells ( $0.3 \%$ of total B cells) were detected, which was similar to the number of $\mathrm{RBD}^{+} \mathrm{MBCs}$ detected using flow cytometry in the recovered group (median $0.23 \%$ ) (Figure 3B). The dynamics of the RBD-specific IgG ASC response was also consistent with the flow cytometry data.

The frequencies of IgA MBC-derived ASCs targeting the Spike and RBD followed a similar pattern, namely, recovered subjects responded more quickly than naïve subjects, but the responses of both groups were roughly comparable at T3. Although the anti-SARS-CoV-2 IgM ASC responses were above the threshold, no significant differences were found between the naive and recovered groups, or between T2 and T3. Thus, our results indicate that in naive recipients, the maximum number of vaccine-induced MBCs is reached only 85 days after the first vaccination, and in terms of kinetics, this process significantly lags behind the formation of serum antigenspecific antibodies. Thus, on day 85 after the first dose of Sputnik $\mathrm{V}$, almost all the vaccinees developed RBD- and S-specific MBCs.

\section{MBC-Derived SARS-CoV-2 Antibody Reactivity}

In most SARS-CoV-2-related studies, virus-binding and virusneutralizing activities are measured in the serum samples, i.e. antibodies secreted by the long-lived plasma cells are primarily assayed. In contrast, we aimed at measuring the quantity and quality of antibodies that will be secreted by MBCs upon ex vivo re-stimulation. To do so, we used 7-day cultures of IL-21/ CD40L-stimulated B cells as a source of MBC-derived antibodies. These conditions mimic the germinal center environment in which B cells differentiate into plasma cells (25). Indeed, approximately $95 \%$ of MBCs become plasmablasts $\left(\mathrm{CD} 19^{+} \mathrm{CD} 20^{-} \mathrm{CD} 27^{\text {hi }} \mathrm{CD} 38^{\text {hi }}\right.$ ) over 7 day culture with CD40L and IL-21 (Figure S5). Virus-specific antibody levels in the culture supernatants were assessed with ELISA on RBD-precoated plates. The stimulated MBCs from all recovered individuals secreted a significant amount of anti-RBD IgG both at T2 and T3 (Figure 4A). In the naïve group at T3, the level of anti-RBD IgG secretion was five- to six-fold reduced compared to that of the recovered group. Stimulated MBCs produced antiRBD IgA and IgM less efficiently than IgG, and the difference between samples from naïve and recovered subjects was less pronounced. We observed an apparent discrepancy between the ELISpot (Figure 3D) and ELISA data for MBC-derived antibodies (Figure 4A). Whereas ELISpot quantifies the secreting cell frequencies in B cell populations, ELISA provides the estimate of a total level of the secreted antibodies, which in turn is the product of the number of ASCs multiplied by the IgG secretion rate. This in turn underlies the importance of using a more comprehensive set of approaches to assess $\mathrm{MBC}$ responses, which in our work includes flow cytometry of RBD-binding circulating cells, ELISpot-based quantification of SARS-CoV-2specific ASC frequencies, and ELISA measurement of the overall levels of MBC-derived antibodies.

Next, we tested the virus-neutralizing activity of MBC-derived antibodies using a pVNT assay. Since the concentration of antibodies in the supernatants was approximately two orders of magnitude lower than that in the plasma, undiluted supernatants 
A

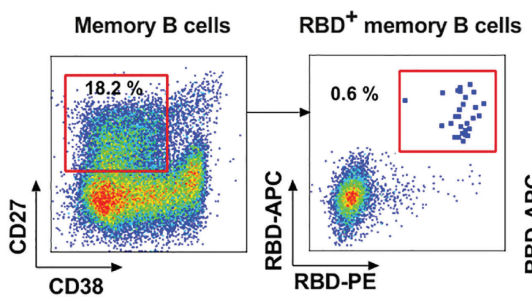

B

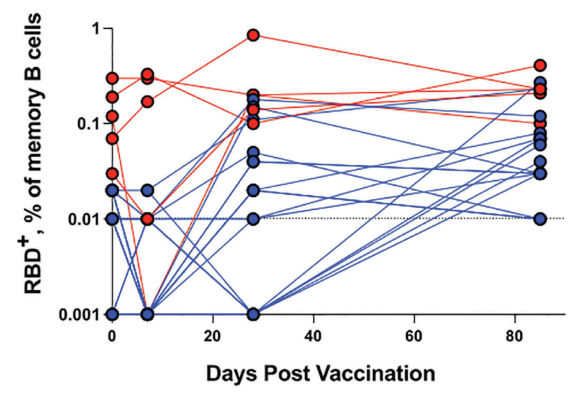

D
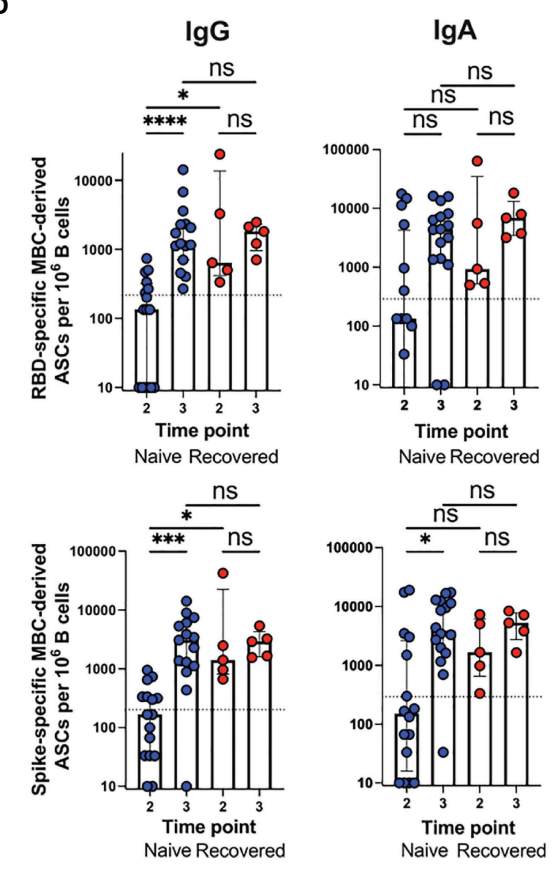

Negative control
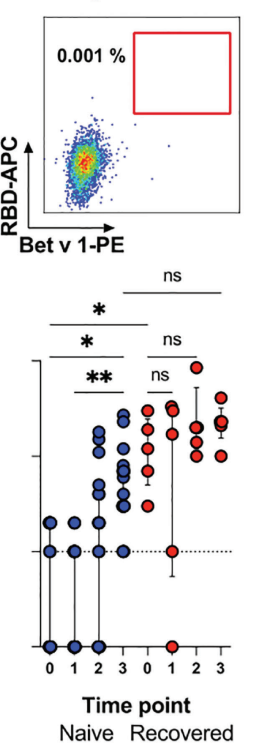

C

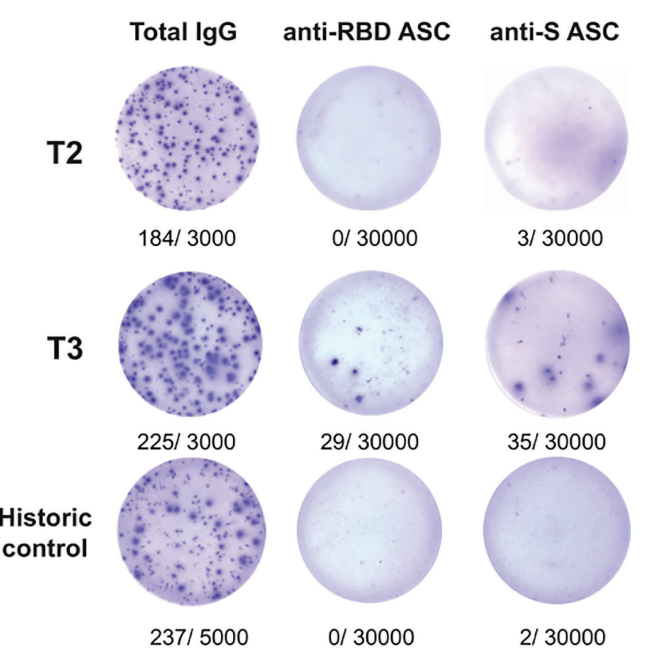

FIGURE 3 | Analysis of the MBC response in Sputnik V-vaccinated individuals. (A) Representative flow cytometry dot plots showing double discrimination of RBD+ MBCs. Numbers inside the plots indicate the percentage of events specific to the respective gates. (B) RBD ${ }^{+} \mathrm{MBCs}$ as a percentage of all memory $\mathrm{B}$ cells $\left(\mathrm{CD} 19^{+} \mathrm{CD} 27^{+} \mathrm{CD}^{-}{ }^{-} \mathrm{lgD}{ }^{-}\right)$. (C) Representative ELISpot showing SARS-CoV-2-specific MBC-derived ASCs. Purified B cells were stimulated with IL-21/CD40L for 7 days and then incubated in ELISpot plates for $16 \mathrm{~h}$ to detect ASCs secreting total (left column), RBD-(middle column) or S-specific (right column) IgG at T2 time point (upper row), T3 (middle row) or in historic control samples (bottom row). The numbers indicated below the wells represent positive dots and the total number of cells in the well. (D) RBD- (upper row) and S-specific (bottom row) MBC-derived ASCs per $10^{6} \mathrm{~B}$ cells from blood samples of naïve $(\mathrm{n}=17$ ) or recovered $(\mathrm{n}=5$ ) vaccinated individuals at different time points. Data for lgG (left column), IgM (middle column) and lgA (right column) ASCs are presented. The dotted lines indicate the threshold for a positive antigen-specific ASC response calculated with pre-pandemic samples per $10^{6} \mathrm{~B}$ cells. Results are shown for individual samples (symbols) from naïve $(n=17)$ and recovered $(n=5)$ recipients. Data are presented as median $\pm I Q R$. Asterisks indicate significant difference between groups determined using the Kruskal-Wallis test, ${ }^{\star} \mathrm{P}<0.05$, ${ }^{\star \star} \mathrm{P}<0.01$, ${ }^{\star \star \star} \mathrm{P}<0.001,{ }^{\star \star \star \star} \mathrm{P}<0.0001$, ns, not significant. ASC, antibody-secreting cell; IQR, interquartile range; RBD, receptorbinding domain. 


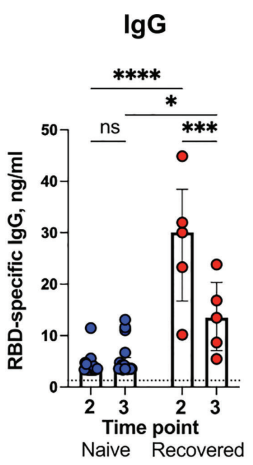

C

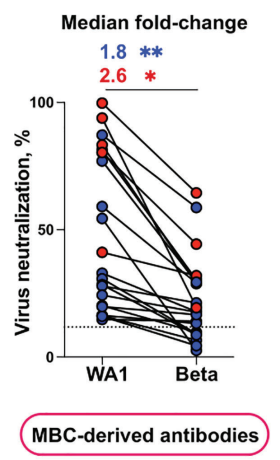

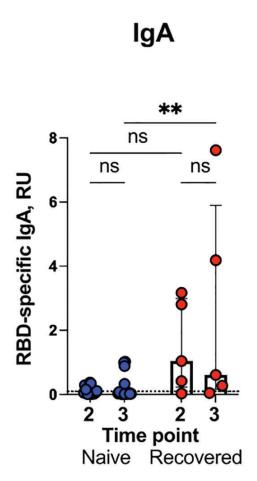

D

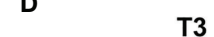

T3

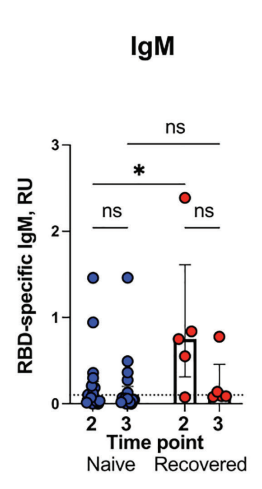

B

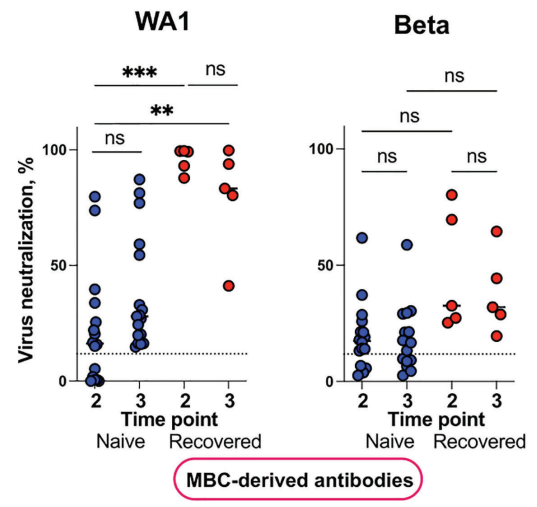

WA1
E

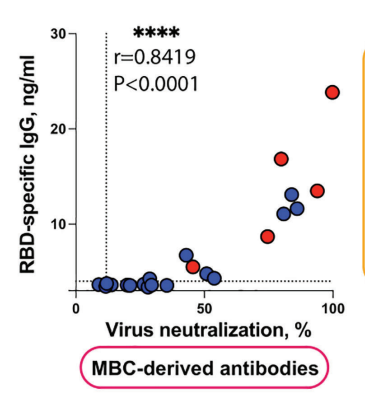

$\mathbf{F}$

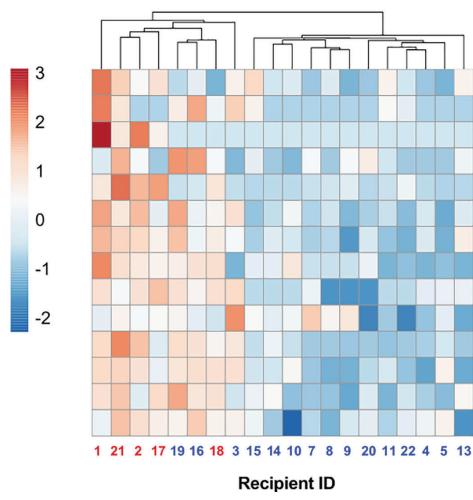

$\mathrm{RBD}^{+}$plasmablasts (T1) $\mathrm{RBD}^{+}$plasmablasts (T2) RBD-specific lgG circulating ASCs (T1) RBD-specific lgG circulating ASCs (T2) Serum anti-RBD IgG (T1) Serum anti-RBD $\lg G(T 3)$ Serum pVNT (WT, T3) $\mathrm{RBD}^{+} \mathrm{MBCs}(\mathrm{T} 2)$ $\mathrm{RBD}^{+} \mathrm{MBCs}(\mathrm{T} 3)$ MBC-derived ASCs (T3) MBC-derived anti-RBD IgG (T3) MBC-derived PVNT (WT,T3) Serum pVNT (Beta, T3) MBC-derived pVNT (Beta,T3)

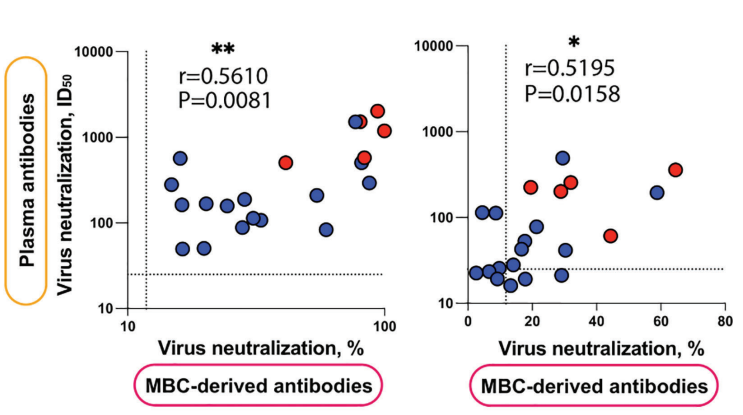

G

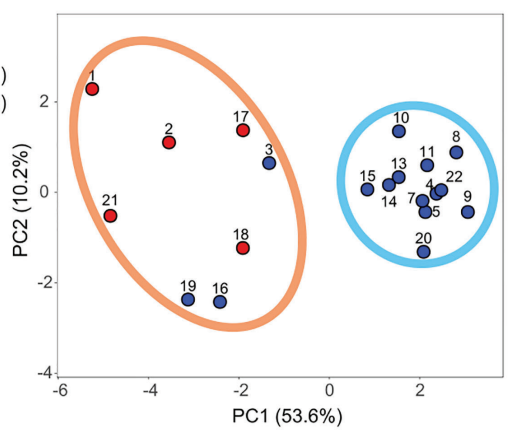

FIGURE 4 | Analysis of MBC-derived antibody response in supernatants of CD4OL/L-21 stimulated B cells from Sputnik V-vaccinated individuals. (A) Production of RBD-specific IgG (left panel), IgA (middle panel) or IgM (right panel) in cultures of IL-21/CD4OL-stimulated B cells from Sputnik V-vaccinated individuals evaluated using ELISA. (B) Virus-neutralizing activity of MBC-derived antibodies against WA1 strain (left panel) and Beta variant (right panel) at T2 and T3 time points.

(C) Paired analysis of virus-neutralizing activity of MBC-derived antibodies against WA1 strain and Beta variant at T3 time point. (D) Spearman's correlation between virus neutralization (\%) and the levels of anti-RBD IgG in supernatants of IL-21/CD4OL-stimulated B cells obtained from Sputnik V-vaccinated individuals at T3 time point. (E) Spearman's correlation between the virus-neutralizing activity of plasma and MBC-derived antibodies. Each symbol represents half-maximal inhibitory plasma dilution $\left(I_{50}\right)$ values and \% of virus neutralization by supernatant of IL-21/CD4OL-stimulated B cells. (F) Heatmap and hierarchical clustering of Sputnik $V$ recipients. Columns denote Sputnik $\vee$ recipients. Rows correspond to immune response variables. Dendrograms on the top illustrate the clustering of Sputnik $\vee$ recipients. Immune response measurement values are color-coded according to the key shown in the upper left. (G) Principal component analysis of Sputnik $V$ recipients. Recipient IDs are shown. Two distinct clusters are indicated by the ovals. Results are shown for individual samples (symbols) from naïve $(n=17)$ and recovered $(n=5)$ recipients. Data are presented as median \pm IQR. Asterisks indicate significant difference between groups determined using the Kruskal-Wallis test, statistics in (C) panel were calculated using Mann-Whitney test. ${ }^{*} \mathrm{P}<0.05,{ }^{* \star} \mathrm{P}<0.01,{ }^{\star \star \star} \mathrm{P}<0.001,{ }^{\star \star \star \star} \mathrm{P}<0.0001$, ns, not significant. IQR, interquartile range; RBD, receptor-binding domain.

were used. First, we ran pVNT assay against the WA1 Spikepseudotyped lentiviral particles. MBC-derived antibodies from recovered individuals inhibited pseudovirus entry in the range of $42-99 \%$ at both time points T2 and T3 (Figure 4B, left panel,
Figure S6). At T2, 10/17 individuals from the naïve group were also responders in the pVNT assay (median neutralization 16.3\%, IQR $1.0-31.8 \%$ ). At T3, all naïve subjects demonstrated virus neutralization above the threshold (Figures 4B, S6). 
Next, we proceeded to measurements of the crossneutralizing potential of $\mathrm{MBC}$-derived antibodies using the Beta variant Spike-pseudotyped lentivirus (Figure 4B, right panel, Figure S6). Similarly to the serum antibodies, MBCderived antibodies displayed weaker cross-neutralization of Beta VOC, which was further reduced in the samples from naïve vaccinees (median fold-change $1.8, \mathrm{P}=0.0066 ; 2.6, \mathrm{P}=$ 0.0317 for naïe and recovered, respectively). Despite the overall reduction in cross-neutralization of the Beta VOC, about half of the vaccinees (15/22) displayed appreciable virus neutralization at T3 (Figure 4C). Also, much as was observed for the serum antibodies, strong correlations were observed between the virusneutralization of $\mathrm{MBC}$-derived antibodies and the level of ex vivo anti-RBD IgG secretion both at T2 (Spearman's $\mathrm{r}=0.9127, \mathrm{P}<$ 0.0001) (Figure S7) and T3 (Spearman's $\mathrm{r}=0.8419, \mathrm{P}<0.0001$ ) (Figure 4D). The virus-neutralizing activity of plasma and MBCderived antibodies at T3 were modestly correlated (Spearman's $\mathrm{r}=$ $0.5610, \mathrm{P}=0.0081 ; \mathrm{r}=0.5195, \mathrm{P}=0.0158$, for WA1 and Beta, respectively) (Figure 4E), pointing at the association between $\mathrm{MBC}$ and long-lived plasma cell responses. Based on these findings, it may be possible to assess the quality of antibodies that MBCs will produce upon re-exposure to an antigen.

We observed that naïve and recovered vaccine recipients differed in several key parameters of humoral and B cell immunity. To investigate if study participants could be divided into subgroups, we carried out hierarchical cluster and principal component analyses. Based on the 14 humoral and B cell response measurements, these analyses, which included 15 naïve and five recovered vaccine recipients indicated the existence of two well-separated clusters (Figures 4F, G). The first compact cluster encompassed exclusively naïve vaccine recipients; the second, somewhat loose cluster included all the recovered and three naïve vaccine recipients. Thus, when several humoral and B cell parameters were taken into consideration, the distinct immune profiles of the naïve and recovered vaccine recipients became clearly apparent. Most interestingly, our comprehensive B cell profiling analysis has uncovered the existence of two categories of vaccine recipients, namely, the high- and low-responders. This highlights the underappreciated heterogeneity of the human immune response to Sputnik V, thereby warranting a systematic identification of predictors and modifiers of this response.

\section{DISCUSSION}

Presently, Sputnik V is used in several countries including Russia, Argentina, India, and Brazil. Nonetheless, the protective properties of this vaccine have been debated and the information available has been somewhat ambivalent $(3-5,15$, $16,26)$. In the present study, a comprehensive analysis of B cell immunity was performed in a cohort of 22 vaccinated subjects, among whom 5 had previously recovered from mild COVID-19. Several parameters were analyzed, namely, (i) the serum antibody titers to RBD, (ii) activity of serum antibodies in the pVNT assay with wild-type SARS-CoV-2 and its mutant variant
Beta B1.351, (iii) detection of RBD-specific plasmablasts and MBCs, (iv) enumeration of circulating and MBC-derived ASCs, and the (v) virus-binding and -neutralizing activity of MBCderived antibodies. Most of these parameters were measured during vaccination with the final time point being 2 months after the first vaccine dose. Even though the study group was rather limited, our in-depth analysis has allowed us to compare the dynamics and magnitude of $\mathrm{B}$ cell immune responses in naïve and COVID-19-recovered vaccine recipients, which reflects the real-world epidemiological situation.

Taken together, our results demonstrate faster and more robust B cell responses to Sputnik V in COVID-19-recovered individuals than in individuals without prior infection. Considering many parameters, recovered individuals achieved the maximum immune response after the first vaccine dose. This is in line with the results obtained in mRNA vaccine studies (1, 23, 27-29). Importantly, we, for the first time, demonstrate this effect at the level of both plasmablasts and MBCs rather than just via quantification of antibody responses and assessment of their neutralization activity. Combined with previously reported data $(5,30)$, our results indicate that a single dose of the adenovirusbased anti-SARS-CoV-2 vaccine, like mRNA-based vaccines, may be sufficient for protective immunity, when used in subjects with pre-existing immunity to SARS-CoV-2.

Among naive vaccine recipients, the response to Sputnik V vaccination was overall slower than that of recovered vaccine recipients, and a fraction of vaccinees who received both doses of Sputnik V never displayed the concentration and neutralization potency of antibodies that would match the levels observed in recovered individuals. Importantly, after the second dose of Sputnik V, the antisera of naïve vaccine recipients showed virusneutralizing activity against the wild-type virus in all but one individual (recipient 22). On average, the antisera from naïve Sputnik V recipients were less potent in terms of their neutralizing ability as compared to the antisera from the mRNA vaccines (31$33)$, yet they were on par with the numbers reported for other adenovirus-based vaccines $(2,34,35)$.

Furthermore, we wanted to investigate if Sputnik V vaccination may efficiently neutralize mutant SARS-CoV-2 variants. Whereas detailed studies are available for the Pfizer and Moderna vaccines, data for Sputnik V are limited. Recently, it was reported that antisera from naïve Sputnik V recipients were 3.1-, 2.8-, and 2.5fold less potent against the Beta, and Delta variants, respectively (15). Another recent study references a median 6.1-fold reduction in the GMT against Beta (4) with a notable comment that when extrapolated to full serum strength, half of the serum samples failed to achieve an $\mathrm{ID}_{80}$ and only one out of 12 achieved an $\mathrm{ID}_{90}$ against Beta. Intriguingly, sera from Sputnik V-vaccinated individuals have been reported to neutralize Omicron variant quite well (36). In this study, we found that the GMT of sera from recovered and naïve recipients exhibited five-fold reduction against Beta compared to that against the wild-type virus in a pVNT assay, but the neutralizing activity of all but one samples was sufficient to achieve an $\mathrm{ID}_{90}$ against when extrapolated to full serum strength. The neutralizing potency of the antisera from recovered subjects against the wild-type variant was more than 
four-fold higher than that of the naive group. Predictably, these samples were also much more active against the Beta variant. This is in excellent agreement with the data reported for mRNA vaccines (1) and is indicative of the higher level of protection against emerging viral variants in the SARS-CoV-2 preexposed vaccinees.

It is interesting to compare the B cell immunity elicited by Sputnik V vaccination with that induced by natural SARS-CoV-2 infection. The dynamics of plasmablast numbers is of special interest because it can be used as a predictor of successful humoral immunity (37). We found low levels of $\mathrm{RBD}^{+}$ plasmablasts and circulating RBD-specific ASCs in vaccinated naive individuals compared to those observed in the acute phase in COVID-19 patients $(20,21)$. Perhaps these differences are associated with the extrafollicular pathway of $\mathrm{B}$ cell activation in the acute phase of COVID-19, which is characterized by massive plasmablast expansion (38). It has been suggested that this pathway may contribute to the pathogenesis of acute COVID19. From this standpoint, the modest plasmablast response observed during vaccination can be viewed as beneficial.

Unlike the plasmablast response, the MBC response was wellpronounced, and the $\mathrm{MBC}$ numbers observed are comparable to those found in acute COVID-19 patients. This is important because vaccine-induced MBCs are known to be central to the longevity of immune memory and are among the first cells to produce massive amounts of antibodies upon antigen reencounter. Although the MBC numbers are informative descriptors of $\mathrm{B}$ cell immunity, the functional activity of the antibodies that will be produced during secondary immune responses is key to our understanding of vaccine-induced protection. To address this question, antigen-specific MBCs were isolated followed by single-cell sequencing of Ig genes and expression of recombinant MBC-derived antibodies $(8,39$, 40). Alternatively, antibody secretion can be induced in cultures of polyclonally stimulated B cells. Seven-day cultures of IL-21/ CD40L-stimulated MBCs isolated from the blood of Sputnik V vaccinees secreted anti-RBD IgG at approximately the same level as MBCs from acute COVID-19 patients. Moreover, MBCderived antibodies from the vast majority of Sputnik V vaccinees could neutralize the ancestral variant of SARS-CoV2 , with antibodies from some of the Sputnik V vaccinees displaying cross-neutralization of the Beta variant.

Virus-neutralizing activity of antibodies is a composite of their quantity and specificity, yet it is also critically dependent on their affinity, which is known to increase during maturation (41). In the acute phase of COVID-19, near-germline clonotypes featuring low mutation numbers are typically formed (38), and no correlation between the activities of plasma and MBC-derived antibodies is observed (20). This is consistent with the idea that MBC and plasma cells may differ in their level of maturation and breadth of reactivity with viral variants (42). In fact, affinity maturation occurs several months following infection or vaccination, and is accompanied with progressive accumulation of up multiple somatic mutations in RBD-specific antibodies (24). Interestingly, two months after vaccination with Sputnik V, modest correlation between the virus-neutralizing activities of plasma and
MBC-derived antibodies was observed in our cohort. This is indicative of the comparable degrees of affinity maturation that the antibodies produced by MBCs and long-lived plasma cells undergo during this period (43).

Two months following vaccination, the differences between the naïve and recovered vaccine recipient cohorts in terms of $\mathrm{MBC}$ numbers and their ability to differentiate into ASCs that secrete RBD-specific and virus-neutralizing antibodies, became less pronounced. Comparison of WA1- and Beta variantneutralizing activities at T3 by serum- and MBC-derived antibodies is indicative of the gradual maturation and continued evolution of the MBC population. Thus, we show that in vitro stimulation of virus-specific MBCs can considerably extend the traditional serological analysis of vaccinated donors. This will allow the study of the dynamics and longevity of antigenspecific MBCs in the course of infection and vaccination. Using this approach, we provide experimental evidence indicating that both recovered and naive vaccinees accumulate similar numbers of virus-specific MBCs at 2 months after the second dose of Sputnik V. Upon antigen stimulation, these cells differentiate into ASCs that secrete virus-specific antibodies, of which a significant proportion is virus-neutralizing. Based on these data, we conclude that the MBCs elicited by Sputnik vaccination, both in their number and productivity, are comparable to those generated during natural infection.

As in the scenario of natural infection, some individuals, classified as low responders, fail to mount robust immune response upon Sputnik V vaccination. The reasons underlying the poor vaccine immunogenicity in these subjects are presently unknown but are likely related to individual features of the immune system. However, our study group was rather limited; 2 months after vaccination, one recipient with the lowest levels of virus-neutralizing antibodies and other indicators of poor B cell immunity developed PCR-confirmed COVID-19 (to be published). Currently, the minimum levels of serum virus-binding and virus-neutralizing activity that are protective against vaccine breakthrough infections remain to be determined. Hence, additional studies are required to establish this minimum level of immunity.

\section{MATERIAL AND METHODS}

\section{Volunteers}

A cohort of 22 Sputnik V recipients was enrolled in December 2020 at the National Research Center Institute of Immunology of The Federal Medical Biological Agency of Russia. None of the participants were pregnant, immunodeficient, or receiving immunosuppressive treatment. Subjects were immunized by intramuscular injection into the deltoid muscle with a 21-day interval between the doses. All subjects received two doses of Gam-COVID-Vac (Sputnik V) vaccine. None of the volunteers had experienced serious adverse events after vaccination. Written informed consent was obtained from each of the study participants before performing any study procedures. The study protocol was reviewed and approved by the Medical Ethical Committee of Institute of Immunology (\#12-1, December 29, 2020). 


\section{Blood Sample Collection and Processing}

Whole-blood samples were collected into heparinized vacutainer tubes (Sarstedt, Cat. No. 04.1927) four times: one day before vaccination, on day 7 after the first and the second doses of vaccine, and on day 85 from the start of vaccination (T0, T1, T2, and T3 time points, respectively) (Figure S1). PBMCs were isolated by density gradient centrifugation. Plasma samples were stored at $-80^{\circ} \mathrm{C}$. B cells were purified from PBMCs by negative selection using the Dynabeads Untouched human B cells kit (Thermo Fisher Scientific, Cat. No. 11351D).

Immunomagnetically separated B cells were cultured in complete DMEM/F12 medium (Cat. No. C470p) supplemented with 10\% FBS (Cat. No. SV30160.03), 2 mM L-glutamine (Cat. No. F032), $24 \mu \mathrm{g} / \mathrm{mL}$ gentamicin (Cat. No. A011p), $1 \mathrm{mM}$ sodium pyruvate (Cat. No. F023), and 10 mM HEPES (Cat. No. F134) (all from Paneko). To obtain MBC-derived antibody-secreting cells (ASCs), B cells were stimulated with $25 \mathrm{ng} / \mathrm{mL}$ interleukin-21 (IL21; PeproTech, Cat. No. 200-21) in the presence of mitomycintreated feeder A549 cells stably expressing CD40L (A549-CD40L, $1 \times 10^{5}$ cells/well) for 7 days at a density of $5 \times 10^{3} \mathrm{~B}$ cells/well in 96-well plates at $37^{\circ} \mathrm{C}$ in $5 \% \mathrm{CO}_{2}$. Stimulated $\mathrm{B}$ cells were harvested 7 days later and used in ELISpot assay. In parallel, supernatants from IL-21/CD40L-stimulated B cells were also collected on day 7 of co-culture for measuring the levels of secreted antibodies in ELISA or virus neutralization assays.

\section{ELISA}

The level of SARS-CoV-2 receptor binding domain (RBD)specific antibodies was measured using ELISA Quantitation Kit (Xema Co., Cat. No. K153G). Plasma samples from vaccinated individuals or supernatants from IL-21/CD40L stimulated B cells were 2-fold serially diluted from 1:20 to $1: 12500$ and 1:2 to 1:200 respectively in blocking buffer. Plates were incubated with samples for 1 hour at room temperature. After washing, the plates were additionally incubated for 1 hour with anti-human IgG, IgM or IgA secondary antibody conjugates with horseradish peroxidase (Jackson Immuno Research, Cat. No. 109-036-088, 109-035-129, and 109-035-011) diluted 1:5,000 in blocking buffer. ELISA plates were washed 7 times and developed for 10 min with $100 \mu \mathrm{L}$ of TMB chromogen solution. The reaction was stopped by adding $50 \mu \mathrm{l} 1 \mathrm{M} \mathrm{H}_{2} \mathrm{SO}_{4}$ and optical density at $450 \mathrm{~nm}$ was measured using the iMark microplate absorbance reader (Bio-Rad, Cat. No. 1681130). Each sample was measured in triplicate. To determine the concentration of IgG, a serial dilution of anti-SARS-CoV-2 RBD-specific human monoclonal antibody iB12 was included on each plate, a calibration curve was built and IgG levels were calculated $(\mu \mathrm{g} / \mathrm{mL})$. When determining the levels of IgM and IgA, we used high-titer convalescent serum as a standard and antibody levels were expressed as relative units (RU).

\section{Flow Cytometry}

Freshly isolated PBMCs were stained with the following antibodies: CD3 FITC (clone TB3), CD16 FITC (clone LNK16), CD19 PE (clone LT19), CD27 PECy5.5 (clone LT27), CD38 PECy7 (clone LT38) [all were produced in-house earlier
(20)]; CD14 FITC (clone MEM-15, Exbio, Cat. No. ED7028); anti-human IgG APC (clone M1310G05, Biolegend Cat. No. 410720) and anti-human IgM APC-Fire750 (clone MHM-88, Biolegend Cat. No. 314546). RBD-specific B cells were detected using double staining with phycoerythrin- and allophycocyaninlabelled RBD (RBD-PE and RBD-APC). Production of recombinant $\mathrm{RBD}$ (isolate Wuhan-Hu-1) or Bet $\mathrm{v} 1$ conjugated to PE or APC was described earlier (Byazrova et al., 2021). Cells were analyzed on a CytoFLEX S flow cytometer (Beckman Coulter). Up to $10 \times 10^{6}$ cells were acquired per sample. Data were analyzed using FlowJo Software (version 10.6.1., Tree Star).

\section{ELISPOT Assay}

Quantification of SARS-CoV-2-specific ASCs was performed by enzyme-linked immunosorbent spot (ELISpot) assay as described previously (20). Briefly, sterile clear 96-well Multiscreen HTS Filter Plates with $0.45 \mu \mathrm{m}$ pore size hydrophobic polyvinylidene difluoride membrane (Merck Millipore, Cat. No. MSIPS4510) were stripped with $70 \%$ ethanol for $2 \mathrm{~min}$, washed and coated with $10 \mu \mathrm{g} / \mathrm{mL}$ of recombinant $\mathrm{RBD}$ or native ectodomain $\mathrm{S}$ protein from SARSCoV-2 (isolate Wuhan-Hu-1). In-house production of recombinant SARS-CoV-2 proteins was described earlier (Byazrova et al., 2021). To capture the total immunoglobulin (IgG, IgM or IgA) produced by ASCs, wells were coated with 10 $\mu \mathrm{g} / \mathrm{ml}$ of rabbit anti-human IgG or IgM (R\&D Systems, Cat. No. SELB002, SELB003), or goat anti-human IgA antibodies (SouthernBiotech, Cat. No. 2050-01).

Freshly purified PBMCs or IL-21/CD40L stimulated B cells were used for quantification of circulating ASCs or MBC-derived ASCs, respectively. Cells were resuspended in complete DMEM/ F12 medium and plated at a density of 250000-3000000 of PBMCs or 100-30000 of purified B cells per well in duplicate. After incubation for $16 \mathrm{~h}$ at $37^{\circ} \mathrm{C}, 5 \% \mathrm{CO}_{2}$, the cells were thoroughly removed with washing buffer $(0.05 \%$ Tween 20 in PBS). Isotype-specific ASCs were detected using IgG- or IgMspecific biotinylated rabbit antibodies (R\&D Systems, Cat. No. SELB002, SELB003) or IgA-specific biotinylated goat antibodies (SouthernBiotech, Cat. No. 2052-08).

After five sequential washes with $0.05 \%$ Tween-20/PBS, streptavidin alkaline phosphatase conjugate (R\&D Systems, Cat. No. SEL002) was added at a 1:60 dilution and the plates were further incubated for 2 hours at room temperature. After several washes, the colorimetric reaction was developed by the addition of Substrate Reagent from B Cell ELISpot Development Module (R\&B Systems, Cat. No. SEL002) until clear distinct spots appeared. The reaction was stopped by rinsing the plate with tap water. ELISpot images were acquired using the CTL ImmunoSpot ${ }^{\circledR}$ analyzer (CTL). Spots were counted using ImmunoSpot ${ }^{\circledR}$ software. Wells coated with an irrelevant protein Bet v 1 served as negative controls.

\section{Pseudotyped Virus Neutralization (pVNT) Assay}

For titration of the neutralizing activity of plasma samples, lentiviral particles pseudotyped with the SARS-CoV-2 S- 
protein of the WA1 strain, or Beta VOC were used. Lentiviral particles were produced as follows. HEK293T cells were transfected with plasmids psPax2 (kind gift from Dr. Didier Trono), pLV-eGFP (was a gift from Pantelis Tsoulfas, (Addgene, Cat. No. 36083)), and the pCAGGS-S $\Delta 19$ plasmid encoding wild-type or Beta $S$ protein (see below). Transfection was performed by the calcium phosphate method; 72 hours after transfection, the supernatants were filtered through a $0.45 \mu \mathrm{m}$ filter, concentrated 20-fold on Amicon ${ }^{\circledR}$ Ultra-15 ultrafiltration cells with a $100 \mathrm{kDa}$ cutoff (Merck, Cat. No. UFC910008). Concentrated supernatant was further centrifuged at 20,000 g, $8^{\circ} \mathrm{C}$ for $90 \mathrm{~min}$. The pellet was resuspended in Opti-MEM medium. Then the viral particles were immediately used in neutralization tests or stored at $-70^{\circ} \mathrm{C}$ for no more than a month. Viral yield was quantified using titration on HEK293ThACE2 cells.

Prior to analysis, plasma samples were heated for $30 \mathrm{~min}$ at $56^{\circ} \mathrm{C}$ to inactivate complement. After that, serial two-fold plasma dilutions ranging 1:10 - 1:1280 were prepared in a 96-well plate and 20,000 lentiviral particles were added in an equal volume of Opti-Mem supplemented with $2.5 \%$ of heat-inactivated FBS. Plasma and viral particles were co-incubated for $30 \mathrm{~min}$ at $37^{\circ} \mathrm{C}$, and added to HEK293T-hACE2 cells. 72 hours following transduction, the percentage of transduced cells was measured in the cultures using flow cytometry. Half-maximal inhibitory dilution $\left(\mathrm{ID}_{50}\right)$ was determined by non-linear regression as the serum dilution that neutralized $50 \%$ of the pseudotyped lentivirus.

\section{Cloning of the Beta Spike Variant}

The construct pCAGGS-S $\Delta 19$ carrying a codon-optimized cassette encoding a SARS-CoV-2 Spike protein (identical in the reference Wuhan-Hu-1 and WA1 isolates) lacking 19 C-terminal residues, which has been shown to boost the viral titers (44), has been described (45).To obtain pCAGGS-S $\Delta 19$ _Beta, sets of complementary mutagenic primers ( $27 \mathrm{nt}$ each) centered at the desired site were used to sequentially introduce the individual mutations (L18F, D80A, D215G, del241-243, R246I, K417N, E484K, N501Y, D614G, A701V) into the coding sequence of $\mathrm{S} \Delta 19$. Sequence identity of the resulting plasmid was confirmed by Sanger sequencing.

\section{Statistical Analysis}

Statistical analysis was performed using Graph Pad Prism (version 8.4.3 GraphPad Software, La Jolla California). The Kruskal-Wallis $\mathrm{H}$ test was used for comparison between multiple groups. The Mann-Whitney test was used for comparison between two groups. $\mathrm{P}<0.05$ was considered statistically significant. Calculation of $95 \%$ confidence intervals (CI) was based on the $\mathrm{t}$-distribution of the log-transformed titers, then back-transformed to the original scale. The correlation between two groups was determined by Spearman rank test. A normalized non-linear regression was performed using GraphPad Prism software (Sigmoidal, 4PL). Heatmap generation and principal component analysis were performed with Clustvis (46) using normalized data. Data are presented as median \pm IQR. Asterisks indicate significant difference between groups, ${ }^{\star} \mathrm{P}<0.05,{ }^{* \star} \mathrm{P}<0.01,{ }^{* *} \mathrm{P}<0.001,{ }^{* * *} \mathrm{P}<0.0001, \mathrm{~ns}=$ not significant.

\section{DATA AVAILABILITY STATEMENT}

The original contributions presented in the study are included in the article/Supplementary Material, further inquiries can be directed to the corresponding author.

\section{ETHICS STATEMENT}

The studies involving human participants were reviewed and approved by the Medical Ethical Committee of National Research Center Institute of Immunology of Federal Medical Biological Agency of Russia, Moscow, Russia (\#12-1, December 29, 2020). The patients/participants provided their written informed consent to participate in this study.

\section{AUTHOR CONTRIBUTIONS}

MB, SK, AG, AT, and AF contributed to study design, data collection, data analysis, data interpretation, literature search, and the writing of this report. MB, SK, EA, TB, GE, AC, IK, and AG performed the experiments, contributed to data analysis, and data interpretation. $\mathrm{MB}, \mathrm{EA}$, and AF contributed to individual recruitment. All authors contributed to the article and approved the submitted version.

\section{FUNDING}

This work was supported by the Russian Science Foundation (Project 21-15-00286) and the Russian Fund for Basic Research (20-04-60527).

\section{ACKNOWLEDGMENTS}

The authors thank all the individuals in the study for the kind donation of both their time and biological material. We thank Gaukhar Yusubalieva, Vladimir Baklaushev, Yuri Lebedin and Rudolf Valenta for their kind help with experiments and for providing reagents. Figure 1A and Supplementary Figure 1 were created with the help of BioRender.com.

\section{SUPPLEMENTARY MATERIAL}

The Supplementary Material for this article can be found online at: https://www.frontiersin.org/articles/10.3389/fimmu.2022.840707/ full\#supplementary-material 


\section{REFERENCES}

1. Goel RR, Apostolidis SA, Painter MM, Mathew D, Pattekar A, Kuthuru O, et al. Distinct Antibody and Memory B Cell Responses in SARS-CoV-2 Naïve and Recovered Individuals Following mRNA Vaccination. Sci Immunol (2021) 6:eabi6950. doi: 10.1126/sciimmunol.abi6950

2. Keeton R, Richardson SI, Moyo-Gwete T, Hermanus T, Tincho MB, Benede $\mathrm{N}$, et al. Prior Infection With SARS-CoV-2 Boosts and Broadens Ad26.COV2.S Immunogenicity in a Variant-Dependent Manner. Cell Host Microbe (2021) 29:1611-9.e5. doi: 10.1016/j.chom.2021.10.003

3. Logunov DY, Dolzhikova IV, Shcheblyakov DV, Tukhvatulin AI, Zubkova OV, Dzharullaeva AS, et al. Safety and Efficacy of an Rad26 and Rad5 VectorBased Heterologous Prime-Boost COVID-19 Vaccine: An Interim Analysis of a Randomised Controlled Phase 3 Trial in Russia. Lancet (2021) 397:671-81. doi: 10.1016/S0140-6736(21)00234-8

4. Ikegame S, Siddiquey MNA, Hung C-T, Haas G, Brambilla L, Oguntuyo KY, et al. Neutralizing Activity of Sputnik V Vaccine Sera Against SARS-CoV-2 Variants. Nat Commun (2021) 12:4598. doi: 10.1038/s41467-021-24909-9

5. Rossi AH, Ojeda DS, Varese A, Sanchez L, Gonzalez Lopez Ledesma MM, Mazzitelli I, et al. Sputnik V Vaccine Elicits Seroconversion and Neutralizing Capacity to SARS-CoV-2 After a Single Dose. Cell Rep Med (2021) 2:100359. doi: 10.1016/j.xcrm.2021.100359

6. Davies NG, Abbott S, Barnard RC, Jarvis CI, Kucharski AJ, Munday JD, et al. Estimated Transmissibility and Impact of SARS-CoV-2 Lineage B.1.1.7 in England. Science (2021) 372:eabg3055. doi: 10.1126/science.abg3055

7. Madhi SA, Baillie V, Cutland CL, Voysey M, Koen AL, Fairlie L, et al. Efficacy of the ChAdOx1 Ncov-19 Covid-19 Vaccine Against the B.1.351 Variant. N Engl J Med (2021) 384:1885-98. doi: 10.1056/NEJMoa2102214

8. Dejnirattisai W, Zhou D, Ginn HM, Duyvesteyn HME, Supasa P, Case JB, et al. The Antigenic Anatomy of SARS-CoV-2 Receptor Binding Domain. Cell (2021) 184:2183-2200.e22. doi: 10.1016/j.cell.2021.02.032

9. Wibmer CK, Ayres F, Hermanus T, Madzivhandila M, Kgagudi P, Oosthuysen B, et al. SARS-CoV-2 501y.V2 Escapes Neutralization by South African COVID-19 Donor Plasma. Nat Med (2021) 27:622-5. doi: 10.1038/ s41591-021-01285-x

10. Zhou D, Dejnirattisai W, Supasa P, Liu C, Mentzer AJ, Ginn HM, et al. Evidence of Escape of SARS-CoV-2 Variant B.1.351 From Natural and Vaccine-Induced Sera. Cell (2021) 184:2348-61.e6. doi: 10.1016/j.cell.2021. 02.037

11. Wang Z, Muecksch F, Schaefer-Babajew D, Finkin S, Viant C, Gaebler C, et al. Naturally Enhanced Neutralizing Breadth Against SARS-CoV-2 One Year After Infection. Nature (2021) 595:426-31. doi: 10.1038/s41586-021-03696-9

12. Kustin T, Harel N, Finkel U, Perchik S, Harari S, Tahor M, et al. Evidence for Increased Breakthrough Rates of SARS-CoV-2 Variants of Concern in BNT162b2-mRNA-Vaccinated Individuals. Nat Med (2021) 27:1379-84. doi: 10.1038/s41591-021-01413-7

13. Tegally H, Wilkinson E, Giovanetti M, Iranzadeh A, Fonseca V, Giandhari J, et al. Detection of a SARS-CoV-2 Variant of Concern in South Africa. Nature (2021) 592:438-43. doi: 10.1038/s41586-021-03402-9

14. Garcia-Beltran WF, Lam EC, St. Denis K, Nitido AD, Garcia ZH, Hauser BM, et al. Multiple SARS-CoV-2 Variants Escape Neutralization by VaccineInduced Humoral Immunity. Cell (2021) 184:2372-2383.e9. doi: 10.1016/ j.cell.2021.03.013

15. Gushchin VA, Dolzhikova IV, Shchetinin AM, Odintsova AS, Siniavin AE, Nikiforova MA, et al. Neutralizing Activity of Sera From Sputnik VVaccinated People Against Variants of Concern (VOC: B.1.1.7, B.1.351, P.1, B.1.617.2, B.1.617.3) and Moscow Endemic SARS-CoV-2 Variants. Vaccines (2021) 9:779. doi: 10.3390/vaccines 9070779

16. Vokó Z, Kiss Z, Surján G, Surján O, Barcza Z, Pályi B, et al. Nationwide Effectiveness of Five SARS-CoV-2 Vaccines in Hungary - The HUN-VE Study. Clin Microbiol Infect (2021) S1198-743X(21)00639-X. doi: 10.1016/ j.cmi.2021.11.011

17. Bos R, Rutten L, van der Lubbe JEM, Bakkers MJG, Hardenberg G, Wegmann F, et al. Ad26 Vector-Based COVID-19 Vaccine Encoding a PrefusionStabilized SARS-CoV-2 Spike Immunogen Induces Potent Humoral and Cellular Immune Responses. NPJ Vaccines (2020) 5:91. doi: 10.1038/s41541020-00243-x
18. Wang P, Nair MS, Liu L, Iketani S, Luo Y, Guo Y, et al. Antibody Resistance of SARS-CoV-2 Variants B.1.351 and B.1.1.7. Nature (2021) 593:130-5. doi: 10.1038/s41586-021-03398-2

19. Wrammert J, Smith K, Miller J, Langley WA, Kokko K, Larsen C, et al. Rapid Cloning of High-Affinity Human Monoclonal Antibodies Against Influenza Virus. Nature (2008) 453:667-71. doi: 10.1038/nature06890

20. Byazrova M, Yusubalieva G, Spiridonova A, Efimov G, Mazurov D, Baranov $\mathrm{K}$, et al. Pattern of Circulating SARS-CoV-2-Specific Antibody-Secreting and Memory B-Cell Generation in Patients With Acute COVID-19. Clin Transl Immunol (2021) 10:e1245. doi: 10.1002/cti2.1245

21. Kuri-Cervantes L, Pampena MB, Meng W, Rosenfeld AM, Ittner CAG, Weisman AR, et al. Comprehensive Mapping of Immune Perturbations Associated With Severe COVID-19. Sci Immunol (2020) 5:eabd7114. doi: 10.1126/sciimmunol.abd7114

22. Sanz I, Wei C, Jenks SA, Cashman KS, Tipton C, Woodruff MC, et al. Challenges and Opportunities for Consistent Classification of Human B Cell and Plasma Cell Populations. Front Immunol (2019) 10:2458. doi: 10.3389/ fimmu.2019.02458

23. Ciabattini A, Pastore G, Fiorino F, Polvere J, Lucchesi S, Pettini E, et al. Evidence of SARS-CoV-2-Specific Memory B Cells Six Months After Vaccination With the BNT162b2 mRNA Vaccine. Front Immunol (2021) 12:740708. doi: 10.3389/fimmu.2021.740708

24. Sokal A, Barba-Spaeth G, Fernández I, Broketa M, Azzaoui I, de la Selle A, et al. mRNA Vaccination of Naive and COVID-19-Recovered Individuals Elicits Potent Memory B Cells That Recognize SARS-CoV-2 Variants. Immunity (2021) 54:2893-907.e5. doi: 10.1016/j.immuni.2021.09.011

25. Ding BB, Bi E, Chen H, Yu JJ, Ye BH. IL-21 and CD40L Synergistically Promote Plasma Cell Differentiation Through Upregulation of Blimp-1 in Human B Cells. JI (2013) 190:1827-36. doi: 10.4049/jimmunol.1201678

26. Bucci EM, Berkhof J, Gillibert A, Gopalakrishna G, Calogero RA, Bouter LM, et al. Data Discrepancies and Substandard Reporting of Interim Data of Sputnik V Phase 3 Trial. Lancet (2021) 397:1881-3. doi: 10.1016/S0140-6736 (21)00899-0

27. Ebinger JE, Fert-Bober J, Printsev I, Wu M, Sun N, Prostko JC, et al. Antibody Responses to the BNT162b2 mRNA Vaccine in Individuals Previously Infected With SARS-CoV-2. Nat Med (2021) 27:981-4. doi: 10.1038/ s41591-021-01325-6

28. Krammer F, Srivastava K, Alshammary H, Amoako AA, Awawda MH, Beach KF, et al. Antibody Responses in Seropositive Persons After a Single Dose of SARS-CoV-2 mRNA Vaccine. N Engl J Med (2021) 384:1372-4. doi: 10.1056/ NEJMc2101667

29. Stamatatos L, Czartoski J, Wan YH, Homad LJ, Rubin V, Glantz H, et al. mRNA Vaccination Boosts Cross-Variant Neutralizing Antibodies Elicited by SARS-CoV-2 Infection. Science (2021) eabg9175. doi: 10.1126/ science.abg 9175

30. Collier DA, Ferreira IATM, Kotagiri P, Datir RP, Lim EY, Touizer E, et al. Age-Related Immune Response Heterogeneity to SARS-CoV-2 Vaccine BNT162b2. Nature (2021) 596:417-22. doi: 10.1038/s41586-021-03739-1

31. Anderson EJ, Rouphael NG, Widge AT, Jackson LA, Roberts PC, Makhene M, et al. Safety and Immunogenicity of SARS-CoV-2 mRNA-1273 Vaccine in Older Adults. N Engl J Med (2020) 383:2427-38. doi: 10.1056/NEJMoa2028436

32. Liu Y, Liu J, Xia H, Zhang X, Fontes-Garfias CR, Swanson KA, et al. Neutralizing Activity of BNT162b2-Elicited Serum. N Engl J Med (2021) 384:1466-8. doi: 10.1056/NEJMc2102017

33. Wu K, Werner AP, Koch M, Choi A, Narayanan E, Stewart-Jones GBE, et al. Serum Neutralizing Activity Elicited by mRNA-1273 Vaccine. N Engl J Med (2021) 384:1468-70. doi: 10.1056/NEJMc2102179

34. Folegatti PM, Ewer KJ, Aley PK, Angus B, Becker S, Belij-Rammerstorfer S, et al. Safety and Immunogenicity of the ChAdOx1 Ncov-19 Vaccine Against SARS-CoV-2: A Preliminary Report of a Phase 1/2, Single-Blind, Randomised Controlled Trial. Lancet (2020) 396:467-78. doi: 10.1016/S0140-6736(20) 31604-4

35. Zhu FC, Guan XH, Li YH, Huang JY, Jiang T, Hou LH, et al. Immunogenicity and Safety of a Recombinant Adenovirus Type-5-Vectored COVID-19 Vaccine in Healthy Adults Aged 18 Years or Older: A Randomised, Double-Blind, Placebo-Controlled, Phase 2 Trial. Lancet (2020) 396:479-88. doi: 10.1016/S0140-6736(20)31605-6 
36. Lapa D, Grousova DM, Matusali G, Meschi S, Colavita F, Bettini A, et al. Retention of Neutralizing Response Against SARS-CoV-2 Omicron Variant in Sputnik V Vaccinated Individuals. medRxiv (2022) 01.15.22269335. doi: 10.1101/2022.01.15.22269335

37. Appanna R, Kg S, Xu MH, Toh Y-X, Velumani S, Carbajo D, et al. Plasmablasts During Acute Dengue Infection Represent a Small Subset of a Broader Virus-Specific Memory B Cell Pool. EBioMedicine (2016) 12:178-88. doi: 10.1016/j.ebiom.2016.09.003

38. Woodruff MC, Ramonell RP, Nguyen DC, Cashman KS, Saini AS, Haddad NS, et al. Extrafollicular B Cell Responses Correlate With Neutralizing Antibodies and Morbidity in COVID-19. Nat Immunol (2020) 21:1506-16. doi: 10.1038/s41590-020-00814-z

39. Abayasingam A, Balachandran H, Agapiou D, Hammoud M, Rodrigo C, Keoshkerian E, et al. Long-Term Persistence of RBD+ Memory B Cells Encoding Neutralizing Antibodies in SARS-CoV-2 Infection. Cell Rep Med (2021) 2:100228. doi: 10.1016/j.xcrm.2021.100228

40. Scheid JF, Barnes CO, Eraslan B, Hudak A, Keeffe JR, Cosimi LA, et al. B Cell Genomics Behind Cross-Neutralization of SARS-CoV-2 Variants and SARSCoV. Cell (2021) 184:3205-21.e24. doi: 10.1016/j.cell.2021.04.032

41. Pape KA, Dileepan T, Kabage AJ, Kozysa D, Batres R, Evert C, et al. HighAffinity Memory B Cells Induced by SARS-CoV-2 Infection Produce More Plasmablasts and Atypical Memory B Cells Than Those Primed by mRNA Vaccines. Cell Rep (2021) 37:109823. doi: 10.1016/j.celrep.2021.109823

42. Laidlaw BJ, Ellebedy AH. The Germinal Centre B Cell Response to SARSCoV-2. Nat Rev Immunol (2021) 22:7-18. doi: 10.1038/s41577-021-00657-1

43. Suan D, Sundling C, Brink R. Plasma Cell and Memory B Cell Differentiation From the Germinal Center. Curr Opin Immunol (2017) 45:97-102. doi: 10.1016/j.coi.2017.03.006
44. Johnson MC, Lyddon TD, Suarez R, Salcedo B, LePique M, Graham M, et al. Optimized Pseudotyping Conditions for the SARS-COV-2 Spike Glycoprotein. J Virol (2020) 94:e01062-20. doi: 10.1128/JVI.01062-20

45. Gorchakov AA, Kulemzin SV, Guselnikov SV, Baranov KO, Belovezhets TN, Mechetina LV, et al. Isolation of a Panel of Ultra-Potent Human Antibodies Neutralizing SARS-CoV-2 and Viral Variants of Concern. Cell Discov (2021) 7:96. doi: 10.1038/s41421-021-00340-8

46. Metsalu T, Vilo J. ClustVis: A Web Tool for Visualizing Clustering of Multivariate Data Using Principal Component Analysis and Heatmap. Nucleic Acids Res (2015) 43:W566-70. doi: 10.1093/nar/gkv468

Conflict of Interest: The authors declare that the research was conducted in the absence of any commercial or financial relationships that could be construed as a potential conflict of interest.

Publisher's Note: All claims expressed in this article are solely those of the authors and do not necessarily represent those of their affiliated organizations, or those of the publisher, the editors and the reviewers. Any product that may be evaluated in this article, or claim that may be made by its manufacturer, is not guaranteed or endorsed by the publisher.

Copyright (c) 2022 Byazrova, Kulemzin, Astakhova, Belovezhets, Efimov, Chikaev, Kolotygin, Gorchakov, Taranin and Filatov. This is an open-access article distributed under the terms of the Creative Commons Attribution License (CC BY). The use, distribution or reproduction in other forums is permitted, provided the original author(s) and the copyright owner(s) are credited and that the original publication in this journal is cited, in accordance with accepted academic practice. No use, distribution or reproduction is permitted which does not comply with these terms. 